\title{
Single-Shot Spinal Anesthesia in an Infant Requiring Magnetic Resonance Imaging and Open Lower-Extremity Surgery: A Case Report
}

\author{
Benjamin A. Sanofsky a, b, d, Benjamin V. Jorgensen ${ }^{a}$, Emmett E. Whitaker ${ }^{a}$, \\ Jennifer W. Lisle ${ }^{\mathrm{c}}$, Brian M. Waldschmidt ${ }^{\mathrm{a}}$
}

\begin{abstract}
Spinal anesthesia (SA) is a safe and effective anesthetic technique for lower abdominal and lower extremity surgery in neonates and infants and is associated with an apparent state of sedation. We report the use of single-shot SA in a 6-week-old infant for a combined magnetic resonance imaging and open surgical biopsy of a deep soft tissue lower extremity mass. By leveraging the unique qualities of SA (sedation and surgical blockade), we avoided the need for general anesthesia. To our knowledge, this is the first reported use of single-shot SA for an infant undergoing two procedures in the same day.
\end{abstract}

Keywords: Infant spinal anesthesia; Magnetic resonance imaging; Open lower-extremity surgery

\section{Introduction}

Spinal anesthesia (SA) in children has been a part of pediatric anesthesia practice since the late 1800s [1]. Neonates and infants exhibit hemodynamic and respiratory stability after SA. Furthermore, SA allows for the avoidance of airway manipulation, opioids, and supplemental oxygen inherent to general anesthesia (GA) [2].

The perioperative safety and long-term effects of GA in infants are a topic of continued interest and controversy.

Manuscript submitted June 3, 2020, accepted June 12, 2020

Published online August 6, 2020

aDepartment of Anesthesiology, University of Vermont Larner College of Medicine, Burlington, VT 05401, USA

bepartment of Anesthesiology, University of Vermont Medical Center, Burlington, VT 05401, USA

'Department of Orthopedic Surgery, University of Vermont Larner College of Medicine, Burlington, VT 05401, USA

${ }^{\mathrm{d} C}$ Corresponding Author: Benjamin A. Sanofsky, Department of Anesthesiology, University of Vermont Medical Center, 111 Colchester Avenue, Burlington, VT 05401, USA. Email: Benjamin.Sanofsky@uvmhealth.org

doi: https://doi.org/10.14740/jmc3519
As such, enthusiasm for awake regional techniques has burgeoned in recent years. For non-painful procedures such as magnetic resonance imaging (MRI), "feed and swaddle" techniques have been shown to be an effective alternative to GA or sedation [3]. However, these techniques are not possible when feeding is contraindicated, such as when surgical intervention under GA is anticipated. SA presents a low-risk solution to this problem. In infants, SA is associated with an apparent "sedate state" shortly after administration, possibly as a result of sensory deafferentation $[2,4]$. These sedating properties, along with motor and sensory blockade, provide ideal conditions for diagnostic MRI followed by immediate surgical intervention.

A limitation of SA in this age group is its relatively brief duration of action. Plain isobaric $0.5 \%$ bupivacaine, 0.3 - 0.6 $\mathrm{mg} / \mathrm{kg}$, provides surgical anesthesia for 30 - 180 (mean 80) min [5]. Adjuncts such as epinephrine, morphine, fentanyl, clonidine and neostigmine have been used to extend the duration, with $1 \mu \mathrm{g} / \mathrm{kg}$ clonidine being shown as a safe and effective option in neonatal and pediatric patients [5-7]. The addition of $1 \mu \mathrm{g} / \mathrm{kg}$ clonidine doubled the duration of blockade when compared to isobaric bupivacaine alone without causing clinically significant cardiovascular or respiratory side effects $[6,7]$.

Multiple same-day procedures under a single anesthetic require adequate coordination of care by the involved departments. MRI followed by immediate surgical intervention typically requires the transport and handoff of the patient that is sedated or under GA, increasing risk of complications [8]. Ample pre-procedural planning improves patient safety and enhances both patient satisfaction and institutional efficiency [9].

Here we report the use of single-shot SA in a 6-week-old male infant for a combined MRI and open surgical biopsy of a deep soft tissue lower extremity mass. This approach obviated the need for intravenous anesthetics, inhaled anesthetics, opioids, airway management, and transport of an infant under GA. To our knowledge, this is the first reported use of single-shot SA for an infant undergoing consecutive imaging and surgery.

This manuscript adheres to the applicable Enhancing the Quality and Transparency of Health Research guidelines. Written consent, including Health Insurance Portability and 
Accountability Act authorization, was obtained from the patient's parents for publication of the case report.

\section{Case Report}

A $3.5 \mathrm{~kg}$ 6-week-old male infant born at term was referred for evaluation of a palpable leg mass. Radiographs and ultrasound revealed an enhancing soft tissue mass intimately associated with the proximal fibula, warranting further imaging with MRI prior to open tissue biopsy (Fig. 1a, b). The orthopedic surgeon requested that both procedures be performed at the same time. In discussion with the surgical team, we elected to perform a single-shot SA for both the MRI and open tissue biopsy. With this goal in mind, a concerted effort was made among radiology staff, anesthesiology, and the surgical team to ensure efficiency and coordination, allowing for a single SA to last throughout imaging, transport, and surgery.

A 24-gauge peripheral intravenous (IV) catheter was placed in the hospital's pediatric preoperative area and local anesthetic cream was applied to the patient's lumbar spine before transport to the MRI suite. A transparent bio-occlusive dressing was placed over the applied anesthetic cream and kept in place for $30 \mathrm{~min}$ prior to induction of SA. In the MRI suite, following sterile preparation and draping, lumbar puncture was obtained in the sitting position at L4-5 with a 25-gauge, 1-inch Quincke needle using anatomic landmarks. SA was induced by intrathecal injection of $0.5 \%$ preservative-free isobaric bupivacaine, $0.7 \mathrm{~mL}$, containing clonidine, $4 \mu \mathrm{g}$. Standard American Society of Anesthesiologists monitors were applied and the patient was swaddled and moved to the MRI scanner. No significant hemodynamic or respiratory changes were observed, and imaging was completed uneventfully for a duration of 27 min while the patient slept.

Following imaging and consultation with radiology, the patient was transported to the operating room (OR) for open tissue biopsy guided by the MRI findings. Cefazolin $138 \mathrm{mg}$ and acetaminophen $10 \mathrm{mg} / \mathrm{kg}$ were administered intravenously prior to surgical start. The surgical field was prepared and a tourniquet was inflated to $150 \mathrm{~mm} \mathrm{Hg}$ for a duration of 16 min, with a total OR time of $43 \mathrm{~min}$. The patient remained hemodynamically stable throughout the procedure without significant variance from baseline. No apnea was noted. Overall, 91 min elapsed between spinal injection and departure from the OR, during which time no supplemental oxygen, sedatives, opioids, or vasopressors were required. Following surgery, the patient was brought to the post-anesthesia care unit and discharged home once motor function returned.

Postoperatively, pathological analysis revealed atypical spindle cells suggestive of a primitive myxoid mesenchymal tumor, notable for a gene fusion of MCC-BRAF. Staging workup with positron emission tomography showed localized disease; however, due to the size and location of the mass, neoadjuvant therapy options were reviewed with the goal of reducing tumor size in order to minimize likelihood of morbidity associated with surgical resection. The patient was followed by pediatric oncology on an outpatient basis and started on a 2-month course of trametinib with plans for subsequent imaging to assess for interval change prior to complete resection.
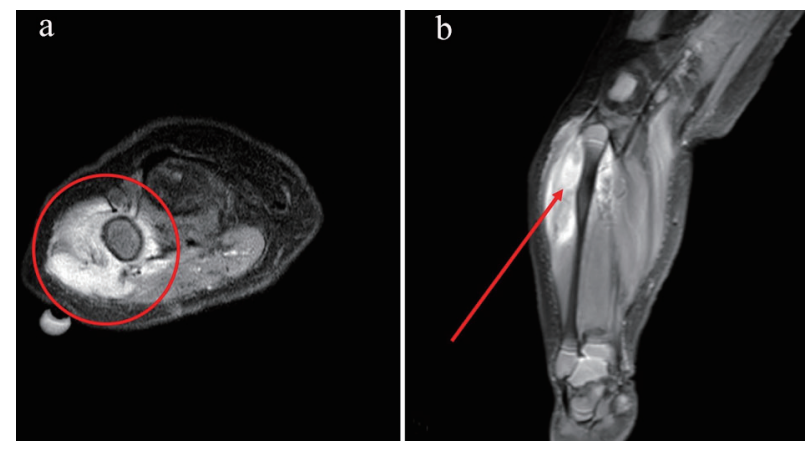

Figure 1. Axial view of soft tissue mass measuring approximately $5.0 \times$ $2.2 \times 2.1 \mathrm{~cm}$ in the lateral aspect of the proximal right calf centered in the peroneus longus muscle, encompassing a portion of the proximal fibula (a). Coronal view of reactive periostitis seen along the posterolateral aspect of the proximal fibula without evidence of osseous infiltration (b).

\section{Discussion}

SA provided an elegant solution to multiple anesthetic considerations given the clinical context of this case. In addition to sensorimotor blockade, we leveraged the "sedate state" associated with infant SA to allow for both MRI and surgical biopsy without GA or supplementary IV sedation.

From a neurodevelopmental standpoint, the literature provides reassuring evidence in regard to the safety of GA in infants [10]. However, ambiguity remains regarding its overall safety and public opinion echoes this uncertainty. In this case, the patient's parents were concerned about the safety of GA and were initially apprehensive about proceeding with any intervention for the mass. Offering an alternative anesthetic not only alleviated concerns around GA's potential long-term effects, but also mitigated some of the known immediate risks associated with GA in infants. GA in this age group is associated with a higher risk of airway complications as well as a higher incidence of cardiac arrest [11]. With SA, the majority of these risks were reduced or eliminated altogether, including potential respiratory complications associated with invasive airway maneuvers [5] and patient transport under GA, which at our institution include changing floors via an elevator.

Providing an anesthetic that allowed for a combined MRI and biopsy was also helpful from an efficiency standpoint. Scheduling the MRI and biopsy separately may have allowed for the MRI to be performed using a feed and swaddle technique but would have delayed diagnosis. Even with the combined MRI and biopsy, it took 1month to receive the final pathology results. Scheduling the MRI and biopsy separately also posed a logistical challenge for the family, who cared for another child and lived several hours away from the medical center, adding complexity to the coordination of the patient's care. By performing both procedures on the same day under SA, both medical and social concerns were addressed.

In planning our anesthetic, one potential limitation of SA that we needed to consider was its duration of effect. Use of SA for lower extremity orthopedic procedures in infants is generally restricted to surgeries lasting less than $90 \mathrm{~min}[12]$. We 
anticipated that the duration of sensory blockade could be inadequate due to the additional time required for the preoperative MRI and the intervening transport. Intrathecal clonidine was added to address this limitation. Interdepartmental coordination also ensured efficiency at each step of the patient's care, improving the likelihood that our single-shot SA would last throughout this combined procedure. The benefits of consistent, prospective planning in administering one continuous anesthetic for multiple procedures in children have been demonstrated in the literature [9], but this practice remains an exception to the norm. Currently, such coordination is performed on an as-needed basis at our institution. To our knowledge, we report the first use of SA for this purpose.

Taking advantage of the unique properties of SA in infants allowed us to provide an optimal anesthetic for this child. Performing the MRI and surgical biopsy in a timely fashion may have improved his chance for a cure of this slow-growing tumor. Our use of SA for this combined procedure addressed the anesthetic considerations raised by a lower extremity mass in a young infant.

\section{Acknowledgments}

None to declare.

\section{Financial Disclosure}

None to declare.

\section{Conflict of Interest}

None to declare.

\section{Informed Consent}

Written consent was obtained from the patient's parents for publication of the case report.

\section{Author Contributions}

BAS and BVJ helped research, write and edit the manuscript; EEW helped write and edit the manuscript; JWL and BMW helped edit the manuscript.

\section{Data Availability}

The authors declare that data supporting the findings of this study are available within the article.

\section{Abbreviations}

GA: general anesthesia; IV: intravenous; MRI: magnetic resonance imaging; OR: operating room; SA: spinal anesthesia

\section{References}

1. Gray HT. A study of spinal anaesthesia in children and infants. The Lancet. 1909;174(4491):913-917.

2. Williams RK, Abajian C. Spinal anesthesia in infants. Techniques in Regional Anesthesia and Pain Management. 1999;3(3):170-176.

3. Heller BJ, Yudkowitz FS, Lipson S. Can we reduce anesthesia exposure? Neonatal brain MRI: Swaddling vs. sedation, a national survey. J Clin Anesth. 2017;38:119122.

4. Hermanns H, Stevens MF, Werdehausen R, Braun S, Lipfert $\mathrm{P}$, Jetzek-Zader M. Sedation during spinal anaesthesia in infants. Br J Anaesth. 2006;97(3):380-384.

5. Gupta A, Saha U. Spinal anesthesia in children: A review. J Anaesthesiol Clin Pharmacol. 2014;30(1):10-18.

6. Kaabachi O, Zarghouni A, Ouezini R, Abdelaziz AB, Chattaoui O, Kokki H. Clonidine $1 \mathrm{microg} / \mathrm{kg}$ is a safe and effective adjuvant to plain bupivacaine in spinal anesthesia in adolescents. Anesth Analg. 2007;105(2):516519.

7. Rochette A, Raux O, Troncin R, Dadure C, Verdier R, Capdevila X. Clonidine prolongs spinal anesthesia in newborns: a prospective dose-ranging study. Anesth Analg. 2004;98(1):56-59, table of contents.

8. Lorinc A, Henson C. All handoffs are not the same: what perioperative handoffs do we participate in and how are they different? APSF Newsletter. October 2017. https:// www.apsf.org/article/all-handoffs-are-not-the-samewhat-perioperative-handoffs-do-we-participate-in-andhow-are-they-different/. Accessed December 12, 2019.

9. Kilkelly JE, Kinch J. An innovative paradigm: coordinating anesthetic care for complex pediatric patients requiring multiple procedures. Journal of Anesthesia \& Clinical Research. 2014;05(11).

10. McCann ME, de Graaff JC, Dorris L, Disma N, Withington D, Bell G, Grobler A, et al. Neurodevelopmental outcome at 5 years of age after general anaesthesia or awakeregional anaesthesia in infancy (GAS): an international, multicentre, randomised, controlled equivalence trial. Lancet. 2019;393(10172):664-677.

11. Cohen MM, Cameron CB, Duncan PG. Pediatric anesthesia morbidity and mortality in the perioperative period. Anesth Analg. 1990;70(2):160-167.

12. Puncuh F, Lampugnani E, Kokki H. Use of spinal anaesthesia in paediatric patients: a single centre experience with 1132 cases. Paediatr Anaesth. 2004;14(7):564-567. 